\title{
Use of population based background rates of disease to assess vaccine safety in childhood and mass immunisation in Denmark: nationwide population based cohort study
}

\author{
(c) $\begin{gathered}\text { (1) (8) } \\ \text { ay }\end{gathered}$
}

\author{
Thomas A Rasmussen resident ${ }^{1}$, Martin R S Jørgensen resident ${ }^{2}$, Stephanie Bjerrum resident ${ }^{3}$, \\ Søren Jensen-Fangel consultant ${ }^{1}$, Henrik Støvring associate professor ${ }^{4}$, Lars Østergaard \\ professor/head ${ }^{1}$, Ole S Søgaard resident ${ }^{1}$
}

'Department of Infectious Diseases, Aarhus University Hospital, DK-8200 Aarhus N, Denmark; ${ }^{2}$ Department of Infectious Diseases, Aalborg Hospital, Aarhus University Hospital, Aalborg, Denmark; ${ }^{3}$ Department of Infectious Diseases, Hvidovre University Hospital, Hvidovre, Denmark; ${ }^{4}$ Department of Public Health, Biostatistics, Aarhus University, Aarhus, Denmark

\begin{abstract}
Objectives To predict the number of selected outcomes temporally associated but not caused by vaccination, to aid causality assessment of adverse events arising after mass immunisation in a paediatric population.

Design Nationwide population based cohort study.

Setting Denmark.

Participants All liveborn infants delivered after 1 January 1980. Study population was followed from date of birth until hospital admission for selected outcome diagnoses, death, first emigration, age 18 years, or 31 December 2009. The study population was subject to vaccines used in standard childhood immunisation in Denmark, with $82-93 \%$ vaccine coverage.

Main outcome measures Incidence of acute infectious and post-infectious polyneuritis (Guillain-Barré syndrome), acute transverse myelitis, optic polyneuritis, facial nerve palsy, anaphylactic shock, seizure, multiple sclerosis, autoimmune thrombocytopenia, type 1 diabetes mellitus, juvenile and rheumatoid arthritis, narcolepsy, and death of unknown cause stratified by sex, age, and season. We predicted the number of events for a hypothetical vaccine cohort of 1000000 people for follow-up periods of up to 182 days.
\end{abstract}

Results The study included 2300227 liveborn infants, yielding 37262
404 person years of follow-up; median follow-up was 16.8 person years.
Incidence of outcome diagnoses spanned from 0.32 per 100000 patient
years for autoimmune thrombocytopenia to 189.82 per 100000 patient
years for seizure. Seasonal differences were most pronounced for
anaphylactic shock, seizure, and multiple sclerosis. Even for rare
outcomes, numerous events were predicted in the hypothetical vaccine
cohort. We predicted that 20 cases of type 1 diabetes mellitus, 19 of
juvenile or rheumatoid arthritis, eight of facial nerve palsy, and five of
multiple sclerosis per 1000 000 children would occur within 42 days
after vaccination.
Conclusions Incorporating exact background rates of disease based
on age, sex, and seasonal distribution could strengthen vaccine safety
assessment, and provides an evidence based focus for discussing the
incremental risk of newly introduced vaccines.
Introduction
Mass immunisation programmes are frequently used in
protecting the population from infectious diseases. The overall
benefit assessment of immunisation programmes includes the
identification and evaluation of possible adverse effects. Since
most serious adverse outcomes rarely occur, scientists are

Extra material supplied by the author (see http://www.bmj.com/content/345/bmj.e5823?tab=related\#webextra)

Web table 1: For children aged $<1$ year: Predicted numbers of outcomes temporally associated with a vaccine dose per 1,000,000 vaccinated subjects

Web table 2: For children aged 1-3 years: Predicted numbers of outcomes temporally associated with a vaccine dose per 1,000,000 vaccinated subjects

Web table 3: For children aged 4-9 years: Predicted numbers of outcomes temporally associated with a vaccine dose per 1,000,000 vaccinated subjects

Web table 4: For children aged 10-17 years: Predicted numbers of outcomes temporally associated with a vaccine dose per 1,000,000 vaccinated subjects 
challenged with identifying these events and determining whether they are causally linked to a specific vaccine or only temporally associated with the vaccine by chance. When new vaccines are introduced on a large scale, passive reporting of adverse drug reactions continues as a postmarketing surveillance system. ${ }^{1}$ However, these systems for passive reporting cannot discriminate events that are temporally associated with vaccine exposure from those that are caused by exposure. Knowing the background rates of relevant diseases would assist in deciding when the number of events exceeds the expected count of non-causally linked events.

The emergence of the H1N1 influenza A virus pandemic in 2009 prompted rapid licensure and broad scale use of H1N1 monovalent and live attenuated vaccines, with ongoing monitoring of potential adverse effects. Children and adolescents were among recommended vaccine recipients in most countries. The Committee for Human Medicinal Products in Europe and the Food and Drug Administration in the United States listed Guillain-Barré syndrome, neuritis, demyelinating disorders, convulsions, anaphylaxis, and Bell's palsy, among others, as adverse events of special interest. ${ }^{2}$ In addition, the 2009 pandemic enhanced efforts to correctly identify background rates of adverse events in the population to which vaccine safety monitoring apply. Several countries including the US, Australia, and countries in Europe have expanded their monitoring systems for vaccine safety. The US first used the Vaccine Adverse Event Reporting System, and then a newer, web based system for active surveillance. Currently, large, population based systems are used for monitoring, including the newly established Post-licensure Rapid Immunization Safety Monitoring project and the Vaccine Safety Datalink project, linking computerised vaccination data with healthcare encounter codes. ${ }^{3}{ }^{5}$

The resulting amount of data for the safety of pandemic H1N1 vaccines among children and adolescents is enormous, but diversity in methods and data presentation has hampered the safety analyses. ${ }^{2}$ Other research has stressed the importance of identifying background rates of selected diseases to distinguish between events temporally occurring and those causally linked to mass vaccination. ${ }^{678}$ Earlier experiences have shown how reports of particular adverse events associated with vaccination can rapidly affect vaccine acceptance. ${ }^{9} 10$

Using unique Danish medical administrative databases, we provide background rates of selected outcomes in a population given standard childhood vaccinations, to help in the causality assessment of adverse events related to a novel vaccine being introduced into a mass immunisation programme.

\section{Methods}

\section{Study design and study population}

This nationwide population based cohort study included all liveborn infants who were delivered in Denmark between 1 January 1980 and 31 December 2009. Study participants were followed from date of birth until the first recorded hospital admission for the earliest date of the following events: one of the selected outcome diagnoses in table $1 \Downarrow$, death, first emigration, age 18 years, or 31 December 2009. The Danish healthcare system provides free, tax supported, medical care for all residents, including all the medical conditions selected for this analysis.

As per routine procedures in the Danish healthcare system, all study participants were subject to standard immunisation through the Danish childhood vaccination programme (table $2 \Downarrow$ ), with a high vaccine coverage (82-93\%). ${ }^{11} 13141516$

\section{Danish Civil Registration System}

The Civil Registration System is a national registry of all Danish residents, which contains information on date of birth, sex, date of migration, and date of death. A 10 digit personal registration number (CPR number) assigned at birth uniquely identifies each person since 1968. The registration system is updated weekly with information on all births, deaths, or emigrations of the population.

\section{Danish National Hospital Registry}

The National Hospital Registry contains information on all patients discharged from non-psychiatric hospitals in Denmark since 1 January 1977. Records for each hospital admission include CPR number, hospital department, inpatient and outpatient discharge diagnoses, and dates of admission and discharge. The treating physician coded diagnoses according to the ICD-8 (international classification of diseases, 8th revision) until the end of 1993, and ICD-10 thereafter.

\section{Identification of outcome diagnoses}

For study participants, we retrieved all hospital discharge diagnoses, including emergency room visits, in the National Health Registry for the following outcomes: acute infectious and post-infectious polyneuritis (Guillain-Barré syndrome), acute transverse myelitis, optic polyneuritis, facial nerve palsy, anaphylactic shock, seizure, multiple sclerosis, autoimmune thrombocytopenia, type 1 diabetes mellitus, juvenile and rheumatoid arthritis, narcolepsy, or death of unknown cause. Table 1 lists the ICD- 8 and ICD-10 codes used for the identification of the outcome events. Most of these diagnoses have been validated in previous reports. ${ }^{17} 18192021$

\section{Statistical analyses}

We calculated the incidence of each diagnosis of interest, ignoring all other diagnoses (excluding death), and if participants were followed to either first admission with the specific diagnosis or end of follow-up. Both primary and secondary diagnoses were retrieved, but in the analyses presented here only primary diagnoses were considered. We reported incidence, stratified by sex and age categories ( $<1,1-3,4-9,10-17$ years), and overall. In analyses considering any diagnoses (any adverse event), we considered only the first occurrence of any of the diagnoses together with end of follow-up. All rates were accompanied by exact $95 \%$ confidence intervals based on the assumption of a Poisson distribution for event counts. We obtained seasonal rates by dividing all events and time at risk into the quarters of the calendar year to which they belonged while ignoring any other information (that is, age, sex, calendar year).

To show the usefulness of these data in the context of a mass immunisation campaign, we constructed a hypothetical vaccine cohort of 1000000 people and computed the predicted count of events for follow-up times of one, seven, 42, and 182 days, respectively, by taking the estimated rate to be the true constant rate over the follow-up period. The corresponding prediction intervals were found by numerical integration of tail probabilities of the relevant Poisson distribution. Web tables 1-4 show similar counts for each age category separately. All statistical analyses were conducted with Stata 11. 


\section{Results \\ Incidence of outcome diagnoses}

The study included 2300227 liveborn infants, yielding a total of 37262404 person years of follow-up; median follow-up time was 16.8 person years. Annual birth counts and annual deaths before age 5 years both declined slightly during the study period (fig $1 \Downarrow$ ). We had no information on rates of influenza vaccine coverage in the study population during the main study period. However, in the period from 2009-10 to 2011-12, influenza vaccine was given to $0.2-0.3 \%$ of children under age 15 , each year. ${ }^{22}$

In total, 73564 incident cases of the selected outcome diagnoses were recorded during the study period among participants younger than 18 years in the Danish population; these cases are presented as age and sex specific incidence (table $3 \Downarrow$ ). There were considerable differences in the incidence of these diagnoses, from 0.32 per 100000 person years for autoimmune thrombocytopenia to 189.82 per 100000 person years for seizure (including febrile seizure). We observed low rates of acute transverse myelitis ( 0.36 per 100000 person years), narcolepsy (0.48), optic polyneuritis $(0.60)$, and Guillain-Barré syndrome (0.67), whereas rates were higher for juvenile and rheumatoid arthritis (16.73) and type 1 diabetes mellitus (17.71).

Incidence of anaphylactic shock, death of unknown cause, multiple sclerosis, and facial nerve palsy ranged from 1.45 to 6.91 per 100000 person years (table 3 ). However, these rates concealed considerable variability in the sex and age distribution. With minor deviations, the incidence of Guillain-Barré syndrome, optic polyneuritis, anaphylactic shock, and type 1 diabetes mellitus increased with age, whereas rates of seizure, multiple sclerosis, autoimmune thrombocytopenia, and death of unknown cause decreased with age. The most common outcome, seizure, had a high incidence of 475.43 per 100000 person years among children younger than 1 year, and a peak in the age group 1-3 years with 593.49 per 100000 person years. Seizure rates then declined with age to 47.24 per 100000 person years for children aged 4-9 years and 30.83 per 100000 person years for those aged 10-17 years (table 3). In a sensitivity analysis of any adverse event that excluded seizures, we saw a different age distribution with stable incidence in the age groups younger than 10 years, but slightly higher rates in those aged 10-17 years.

Some outcomes had seasonal fluctuation, whereas others had stable incidence throughout the year (fig $2 \Downarrow$ ). Seasonal variation was most pronounced for seizures and multiple sclerosis, with higher rates during the winter, and for anaphylactic shock, with an almost threefold higher rate in the third quarter than in the first.

\section{Expected number of outcomes after vaccination}

Table $4 \Downarrow$ shows the predicted number of first time coincident outcomes expected to be temporally associated with a vaccine dose by chance alone in a hypothetical mass immunisation campaign of 1000000 vaccine recipients younger than 18 years. Even for relatively rare outcomes and in the absence of any causal relation with the vaccine, a substantial number of events would be expected to occur shortly after receiving a vaccine dose. For example, 20 cases of type 1 diabetes mellitus, 19 of juvenile or rheumatoid arthritis, eight of facial nerve palsy, and five of multiple sclerosis would be expected to occur within 42 days after vaccination. Within the first $24 \mathrm{~h}$ of vaccination, five cases of seizure would be expected, and 36 and 218 cases of seizure would be expected to occur within seven and 42 days of vaccination, respectively. Death of an unknown cause would be expected to occur twice within 42 days, and nine times within 182 days after receiving a vaccine dose.

The $80 \%$ and $99 \%$ upper boundaries of the predicted number of coincident adverse events provided in table 4 can be used to assess the likelihood that adverse events of a given type are not only temporally associated with a vaccine dose, but also causally associated. For example, there is a $99 \%$ probability that no more than 52 cases of seizure would occur within seven days of a vaccine dose, if no causal association existed (table 4). Since these upper boundaries are only rarely exceeded in the absence of any causal relation, any crossing of these boundaries should be considered a signal of a possible unexpected adverse reaction to the vaccine being tested.

\section{Discussion}

In this study, we followed a large cohort of Danish children to describe the incidence of selected outcome diagnoses in a population given standard childhood vaccination. We found considerable variation in incidence between the different diagnoses, with seizure being the most frequently recorded diagnosis. In a hypothetical vaccine cohort of 1000000 participants younger than 18 years, we predicted that about 44 cases of type 1 diabetes mellitus, juvenile or rheumatoid arthritis, and multiple sclerosis would occur randomly within 42 days of a given immunisation. Because some of the selected outcomes are highly age specific, we also calculated the predicted number of outcomes stratified by age ( $<1,1-3,4-9,10-17$ years), providing data that can be more readily applied within specific age groups (web tables 1-4). Finally, we provided $80 \%$ and $99 \%$ upper boundaries of the predicted number of coincident adverse outcomes that could help in the causality assessment between reported adverse events and a newly introduced vaccine among children and adolescents.

\section{Strengths and limitations of study}

The population based databases in Danish healthcare, with unique identification of each person, aided analyses within a large, uniform population. To our knowledge, this is the largest study to provide incidence of outcomes that are often linked to vaccine safety concerns within a paediatric population. Our extensive data material enabled us to estimate sex and age specific incidence with high precision. In addition, study participants were followed from birth until the first event that terminated follow-up; thus, we excluded virtually any possibility that outcome events could have occurred before inclusion.

We recognise several limitations of our study. Firstly, only the primary hospital discharge diagnoses and emergency room visits were included. Although the diagnoses selected for this analysis were generally handled in the hospital setting, we cannot rule out that some patients were treated exclusively in the primary sector and hence not recorded in our material. However, even for a relatively benign event such as febrile seizure, the completeness of the Danish National Health Registry exceeds $70 \%$ of all traceable hospital and non-hospital cases. ${ }^{19} \mathrm{We}$ did not consider secondary diagnoses in the analyses to avoid registering events linked to other medical conditions that were not the focus of this study. Secondly, we did not review medical charts to validate the accuracy of all the diagnoses included in this analysis; instead, we relied on previous studies in which validation of diagnoses from the National Health Registry were performed. 
Thirdly, our findings should be extrapolated with care to populations of different race, ethnicity, and environment because disease rates may vary. Fourthly, since study participants were followed up to the first recorded admission for one of the selected outcome diagnoses, subsequent events of the same type in the same person were not included in our analyses. This restriction, however, reduced bias from multiple contacts at the same hospital by the same person (for example, owing to a chronic disease such as diabetes). For some of the selected diseases, there may have been a time delay from onset of symptoms to proper diagnosis that could have affected our time estimates. Finally, we described the incidence of selected outcomes in a vaccine exposed population; consequently, extrapolation to other populations should take into account any differences in childhood vaccination programmes and vaccine coverage.

\section{Comparison with other studies}

Other studies have made similar use of the Danish healthcare databases to assess a causal relation between vaccination and adverse events. Adverse reactions from the measles, mumps, and rubella (MMR) vaccine were investigated in a study that applied a cohort based, data mining methodology to compare the observed incidence with the expected incidence of adverse events in different time periods relative to immunisation. ${ }^{23}$ This method showed promise, but detection bias arising from immunisation given as part of a medical visit would imply causal associations between immunisation and diseases that could, in reality, primarily be associated with the medical screening procedure. Thus, this factor needs to be controlled for. ${ }^{23}$

We described the background rates of a number of selected outcomes rather than investigating adverse events after a specific vaccine. Our data can therefore be applied in the assessment of various vaccine interventions, but should be subjected to the same careful considerations regarding sources of bias when medical inspection is performed as part of immunisation. Rates of autoimmune diseases have also been estimated among members of the Kaiser Permanente health plan for use in studies of adverse events after vaccination. ${ }^{24}$ Furthermore, in a study similar to ours, data for background rates of possible outcomes in people after receiving the pandemic $\mathrm{H} 1 \mathrm{~N} 1$ influenza vaccine were identified for several countries and presented conjointly. ${ }^{7}$ Our study differed from these reports in several ways. We focused on the paediatric population to provide estimates with high precision within the demographic group that is most often immunised. In addition, the population based data sources used were not subject to the same selection bias as cohort data from a private healthcare organisation. We obtained diagnostic information in a large, uniform population with extensive follow-up and were therefore able to provide estimates with high precision. Finally, we investigated the effect of seasonality on the incidence of the different outcomes.

Mass immunisation of large population groups, such as those during the H1N1 influenza A pandemic or when new vaccines are introduced into the childhood immunisation programme, often cause safety concerns when adverse medical events are reported in a temporal association with vaccination. Even sporadic reports of adverse events might compromise public confidence and reduce vaccine acceptance, often to the disadvantage of public health. When a study claimed an association between the MMR vaccine and risk of autism, ${ }^{9}$ it negatively affected vaccine uptake and coincided with subsequent measles outbreaks. ${ }^{10}$ Epidemiological and mechanistic studies have since favoured the rejection of a causal association between MMR and autism. ${ }^{25} 262728$
Available rates for sudden death in adolescents made it possible to continue the vaccine programme for human papilloma virus in Austria, even though a teenage athlete died shortly after introduction of the vaccine. ${ }^{29}$ Also, during the 2006 seasonal influenza vaccination in Israel, the programme was halted because of the death of four people within $24 \mathrm{~h}$ after receiving the vaccine. The four cases were all patients at high risk of sudden death, and post-event analysis showed that an even higher number of events within this patient group were expected to occur within $24 \mathrm{~h}$ of vaccination. ${ }^{30}$

The fast implementation of the vaccines used against the H1N1 influenza pandemic was unprecedented on a global scale and evoked anticipated concerns of unknown adverse effects. Numerous studies have since raised or dealt with the apprehension that this vaccine could be associated with Guillain-Barré syndrome, ${ }^{31} 3233$ transverse myelitis, ${ }^{35} 36$ narcolepsy in children, ${ }^{37}{ }^{38}$ retrograde amnesia, ${ }^{40}$ or other adverse events. ${ }^{2} 33414$ However, such analyses usually cannot be produced in time to respond to the rapidly evolving safety concern when reports of vaccine related adverse events spread quickly throughout society by the internet and mass media, leading to the loss of public confidence.

\section{Conclusions and policy implications}

A complex mix of scientific, economic, psychological, sociocultural, and political factors drives public decision making related to vaccine acceptance, of which transparency and honesty of health authorities are major determinants of public trust. ${ }^{43}$ High quality estimates of the expected number of medical events that will be temporally associated with a vaccine dose during mass immunisation enable health authorities to deal with some of the safety concerns by carefully explaining the anticipated nature of temporally associated events. Thus, the results of our study are of general interest well beyond public health systems and officials, because it provides a foundation for the safety assessment and subsequent public information when new vaccine formulations are introduced against new diseases or added to the childhood immunisation schedule. By incorporating information on background rates of rare outcomes, general practitioners would be in a better position to guide recipients of new vaccines and have their arguments strengthened when confronted with events interpreted by patients or anti-vaccine groups as being caused by vaccination. In areas with public distrust and low vaccine acceptance, this information provides an evidence based focus for discussing the risks and benefits of newly introduced vaccines.

Previous studies have shown the limitations of spontaneous reports with respect to the assessment of causality and emphasised the importance of timely available background event rates in the study of adverse events after immunisation. ${ }^{44}$ As suggested elsewhere, the development of electronic archives with information on background rates of relevant diseases that can be readily applied in the local context would strengthen the assessment of vaccine safety and causality evaluation. ${ }^{7}$ Health delivery systems with strong health information structures relying on population based registries are best equipped for this challenge. We believe that our study, and those of others, ${ }^{6723243145}$ can provide grounds on which to build such efforts.

Contributors: TAR and MRSJ have joint first authorship, performed data interpretation, and drafted the manuscript. HS planned and performed statistical analyses, and revised the manuscript critically for important intellectual content. OSS and $L \varnothing$ conceived of the study and design, performed data collection and data interpretation, and revised the 


\section{What is already known on this topic}

Few studies have examined background incidence of diagnoses often associated with vaccine safety concerns in order to assess the causality of serious adverse events during immunisation

No comprehensive population based study has been performed to describe such incidence among children and adolescents

\section{What this study adds}

Unique Danish medical registries have allowed population based sampling over a prolonged period, to provide age and sex specific incidence of selected diagnoses primarily of autoimmune genesis among children and adolescents

These data could help distinguish events temporally associated with vaccine exposure from those events caused by such exposure In areas with public distrust and low vaccine acceptance, this information provides an evidence based focus for discussing the risks and benefits of newly introduced vaccines

manuscript critically for important intellectual content. SB and SJ-F participated in data interpretation and revised the manuscript critically for important intellectual content. All authors gave final approval for the version to be published, had full access to all of the data (including statistical reports and tables) in the study, and can take responsibility for the integrity of the data and the accuracy of the data analysis.

Funding: The study was funded by the Department of Infectious Diseases, Aarhus University Hospital, Denmark.

Competing interests: All authors have completed the Unified Competing Interest form at www.icmje.org/coi_disclosure.pdf (available on request from the corresponding author) and declare: support from Aarhus University Hospital for the submitted work; no financial relationships with any organisations that might have an interest in the submitted work in the previous 3 years; no other relationships or activities that could appear to have influenced the submitted work.

Ethical approval: The Danish Data Protection Agency approved the establishment of the cohort and the linkage between the registries in this study (J number 2010-41-5602). The study was not subject to approval by the ethics committee because data collection did not involve direct patient contact and all data were fully de-identified.

Data sharing: Technical appendix, statistical code, or dataset available from the corresponding author at thomrasm@rm.dk.

1 Berlin JA, Glasser SC, Ellenberg SS. Adverse event detection in drug development: recommendations and obligations beyond phase 3. Am J Public Health 2008:98:1366-71.

2 Wijnans L, de Bie S, Dieleman J, Bonhoeffer J, Sturkenboom M. Safety of pandemic H1N1 vaccines in children and adolescents. Vaccine 2011;29:7559-71.

3 Destefano F, Tokars J. H1N1 vaccine safety monitoring: beyond background rates. Lancet 2010;375:1146-7.

4 Lieu TA, Kulldorff M, Davis RL, Lewis EM, Weintraub E, Yih K, et al. Real-time vaccine safety surveillance for the early detection of adverse events. Med Care 2007;45(10 supp 2):S89-95.

5 Salmon DA, Akhtar A, Mergler MJ, Vannice KS, Izurieta H, Ball R, et al. Immunization-safety monitoring systems for the $2009 \mathrm{H} 1 \mathrm{~N} 1$ monovalent influenza vaccination program. Pediatrics 2011;127(suppl 1):S78-86.

6 Siegrist CA, Lewis EM, Eskola J, Evans SJ, Black SB. Human papilloma virus immunization in adolescent and young adults: a cohort study to illustrate what events might be mistaken for adverse reactions. Pediatr Infect Dis J 2007;26:979-84.

7 Black S, Eskola J, Siegrist CA, Halsey N, Macdonald N, Law B, et al. Importance of background rates of disease in assessment of vaccine safety during mass immunisation with pandemic H1N1 influenza vaccines. Lancet 2009;374:2115-22.

8 Huang WT, Chuang JH, Kuo SH. Monitoring the safety of pandemic $\mathrm{H} 1 \mathrm{~N} 1$ vaccine. Lancet 2010;375:1164.

9 Wakefield AJ. MMR vaccination and autism. Lancet 1999;354:949-50.

10 Jansen VA, Stollenwerk N, Jensen HJ, Ramsay ME, Edmunds WJ, Rhodes CJ. Measles outbreaks in a population with declining vaccine uptake. Science 2003;301:804

11 Statens Serum Institut. DTaP-IPV/Hib vaccination coverage 2010. 2011. www.ssi.dk/ English/News/EPI-NEWS/2011/No\%2020\%20-\%202011.aspx.

12 Statens Serum Institut. HPV vaccination, coverage 2010. 2011. www.ssi.dk/English/News EPI-NEWS/2011/No\%2018\%20-\%202011.aspx.

13 Statens Serum Institut. MMR vaccination coverage 2010. 2011. www.ssi.dk/English/News/ EPI-NEWS/2011/No\%2021\%20-\%202011.aspx.

14 Statens Serum Institut. PCV coverage and invasive pneumococcal disease 2010. 2011. www.ssi.dk/English/News/EPI-NEWS/2011/No\%2019\%20-\%202011.aspx.

15 Hviid A, Melbye M. Measles-mumps-rubella vaccination and asthma-like disease in early childhood. Am J Epidemiol 2008;168:1277-83.

16 Sun Y, Christensen J, Hviid A, Li J, Vedsted P, Olsen J, et al. Risk of febrile seizures and epilepsy after vaccination with diphtheria, tetanus, acellular pertussis, inactivated poliovirus, and Haemophilus influenzae type B. JAMA 2012;307:823-31.

17 Nørgaard M JJ, Gradus J, Lash TL, Sørensen HT. Problems with onset of multiple sclerosis based on hospital discharge data (abstract). Pharmacoepidemiol Drug Saf2007:16:S220-1.

18 Christiansen CF, Christensen S, Farkas DK, Miret M, Sorensen HT, Pedersen L. Risk of arterial cardiovascular diseases in patients with multiple sclerosis: a population-based cohort study. Neuroepidemiology 2010;35:267-74.
19 Vestergaard M, Obel C, Henriksen TB, Christensen J, Madsen KM, Ostergaard JR, et al The Danish National Hospital Register is a valuable study base for epidemiologic research in febrile seizures. J Clin Epidemiol 2006;59:61-6.

20 Thomsen RW, Hundborg HH, Lervang HH, Johnsen SP, Sorensen HT, Schonheyder HC. Diabetes and outcome of community-acquired pneumococcal bacteremia: a 10-year population-based cohort study. Diabetes Care 2004:27:70-6.

21 Norgaard M, Jensen AO, Engebjerg MC, Farkas DK, Thomsen RW, Cha S, et al. Long-term clinical outcomes of patients with primary chronic immune thrombocytopenia: a Danish population-based cohort study. Blood 2011;117:3514-20

22 Statens Serum Institut. [Vaccination surveillance]. www.ssi.dk/Smitteberedskab/ Sygdomsovervaagning/VaccinationSurveillance. aspx?vaccination $=14 \&$ season $=2009 \%$ $7 \mathrm{C} 2011$ \&sex $=3$ \&agegroup $=10 \&$ landsdel $=100 \&$ kommune $=1000 \&$ axis $=$ AgeGroup \& yaxis=Total\&show $=$ Table\&datatype $=$ Vaccination\#HeaderText.

23 Svanstrom H, Callreus T, Hviid A. Temporal data mining for adverse events following immunization in nationwide Danish healthcare databases. Drug Saf 2010;33:1015-25.

24 Klein NP, Ray P, Carpenter D, Hansen J, Lewis E, Fireman B, et al. Rates of autoimmune diseases in Kaiser Permanente for use in vaccine adverse event safety studies. Vaccine 2010;28:1062-8.

25 Madsen KM, Vestergaard M. MMR vaccination and autism: what is the evidence for a causal association? Drug Saf 2004;27:831-40

26 Madsen KM, Hviid A, Vestergaard M, Schendel D, Wohlfahrt J, Thorsen P, et al. A population-based study of measles, mumps, and rubella vaccination and autism. N Engl J Med 2002;347:1477-82.

27 Honda H, Shimizu Y, Rutter M. No effect of MMR withdrawal on the incidence of autism: a total population study. J Child Psychol Psychiatry 2005;46:572-9.

28 Institute of Medicine. Adverse effects of vaccines-evidence and causality. The National Academies Press, 2010.

29 Lower J. [Two unclear cases of death. Can we still recommend HPV vaccination?]. MMW Fortschr Med 2008;150:6.

30 Kokia ES, Silverman BG, Green M, Kedem H, Guindy M, Shemer J. Deaths following influenza vaccination-background mortality or causal connection? Vaccine 2007:25:8557-61.

31 Deeks SL, Lim GH, Simpson MA, Rosella L, Mackie CO, Achonu C, et al. Estimating background rates of Guillain-Barre Syndrome in Ontario in order to respond to safety concerns during pandemic $\mathrm{H} 1 \mathrm{~N} 1 / 09$ immunization campaign. BMC Public Health 2011;11:329.

32 Dieleman J, Romio S, Johansen K, Weibel D, Bonhoeffer J, Sturkenboom M. Guillain-Barre syndrome and adjuvanted pandemic influenza A (H1N1) 2009 vaccine: multinational case-control study in Europe. BMJ 2011;343:d3908.

33 Williams SE, Pahud BA, Vellozzi C, Donofrio PD, Dekker CL, Halsey N, et al. Causality assessment of serious neurologic adverse events following $2009 \mathrm{H} 1 \mathrm{~N} 1$ vaccination. Vaccine 2011.

34 Choe YJ, Cho H, Bae GR, Lee JK. Guillain-Barre syndrome following receipt of influenza A (H1N1) 2009 monovalent vaccine in Korea with an emphasis on Brighton Collaboration case definition. Vaccine 2011;29:2066-70.

35 Arcondo MF, Wachs A, Zylberman M. [Transverse myelitis associated with anti-influenza A (H1N1) vaccination]. Medicina (B Aires) 2011;71:161-4.

36 Sato $\mathrm{N}$, Watanabe $\mathrm{K}$, Ohta K, Tanaka $\mathrm{H}$. Acute transverse myelitis and acute motor axona neuropathy developed after vaccinations against seasonal and $2009 \mathrm{~A} / \mathrm{H} 1 \mathrm{~N} 1$ influenza. Intern Med 2011;50:503-7.

37 Adjuvant-containing $\mathrm{H} 1 \mathrm{~N} 1$ influenza vaccine pandemrix: narcolepsy in children. Prescrire Int 2011;20:154.

38 Bardage C, Persson I, Ortqvist A, Bergman U, Ludvigsson JF, Granath F. Neurological and autoimmune disorders after vaccination against pandemic influenza $A(H 1 N 1)$ with a monovalent adjuvanted vaccine: population based cohort study in Stockholm, Sweden. BMJ 2011:343:d5956.

39 Haba-Rubio J, Rossetti AO, Tafti M, Heinzer R. [Narcolepsy with cataplexy associated with $\mathrm{H} 1 \mathrm{N1}$ vaccination]. Revue neurologique 2011;167:563-6.

40 Mitrakrishnan S, Ranjanie G, Thirunavakarasu T, Manjula C, Nayananjani K. Seizures and retrograde amnesia with cerebrospinal fluid changes following $\mathrm{H} 1 \mathrm{~N} 1$ influenza vaccination. Vaccine 2011;29:6369-70.

41 Wu J, Xu F, Lu L, Lu M, Miao L, Gao T, et al. Safety and effectiveness of a 2009 H1N1 vaccine in Beijing. N Engl J Med 2010;363:2416-23.

42 Yin JK, Khandaker G, Rashid H, Heron L, Ridda I, Booy R. Immunogenicity and safety of pandemic influenza A (H1N1) 2009 vaccine: systematic review and meta-analysis. Influenza Other Respi Viruses 2011;5:299-305.

43 Larson HJ, Cooper LZ, Eskola J, Katz SL, Ratzan S. Addressing the vaccine confidence gap. Lancet 2011;378(9790):526-35.

44 Folkenberg M, Callreus T, Svanstrom H, Valentiner-Branth P, Hviid A. Spontaneous reporting of adverse events following immunisation against pandemic influenza in Denmark November 2009-March 2010. Vaccine 2011;29:1180-4.

45 Jiang GX, Cheng Q, Link H, de Pedro-Cuesta J. Epidemiological features of Guillain-Barre syndrome in Sweden, 1978-93. J Neurol Neurosurg Psychiatry 1997;62:447-53.

Accepted: 21 August 2012 
Cite this as: BMJ 2012;345:e5823

This is an open-access article distributed under the terms of the Creative Commons Attribution Non-commercial License, which permits use, distribution, and reproduction in any medium, provided the original work is properly cited, the use is non commercial and is otherwise in compliance with the license. See: http://creativecommons.org/licenses/bync/2.0/ and http://creativecommons.org/licenses/by-nc/2.0/legalcode. 


\section{Tables}

Table 1 | Codes used for identification of selected outcomes from the International Classification of Diseases (ICD)

\begin{tabular}{lcc} 
Diagnoses & ICD-8 & ICD-10 \\
Acute infectious and post-infectious polyneuritis (Guillain-Barré syndrome) & 34400 & G61.0 \\
\hline Acute transverse myelitis & Missing & G36.0, G37.0, G37.8, G37.9 \\
\hline Optic polyneuritis & 34100 & H46 \\
\hline Facial nerve palsy & 35099 & G51.0 \\
\hline Anaphylactic shock & $99949-99959$ & T78.2 \\
\hline Seizure & 7802 & R56.0, R56.8 \\
\hline Multiple sclerosis & 340 & G35 \\
\hline Autoimmune thrombocytopenia & 28710 & D69.4 \\
\hline Type 1 diabetes mellitus & 249 & E10 \\
\hline Juvenile and rheumatoid arthritis & $71209,71219-71239,71259$ M05, M06, M080, M082-M084, M088, M089 \\
\hline Narcolepsy & $3470 x$ & G47.4 \\
\hline Death of unknown cause & 795 & R95, R96.0, R96.1, R98 \\
\hline
\end{tabular}


Table 2| Historical overview of the Danish childhood vaccination programme

\begin{tabular}{lc} 
Vaccine & Vaccination period \\
Single component pertussis & $1969-96$ \\
\hline Diphtheria-tetanus-polio & $1969-96$ \\
\hline Oral poliomyelitis vaccine & $1960-2003$ \\
\hline Measles-mumps-rubella & $1987-$ \\
\hline Single component Hib & $1993-2002$ \\
\hline Diphtheria-tetanus revaccination at age 5 years & $1996-2003$ \\
\hline Diphtheria-tetanus-pertussis-polio & $1997-2002$ \\
\hline Diphtheria-tetanus-pertussis-polio/Hib & $2002-$ \\
\hline Diphtheria-tetanus-pertussis-revaccination & $2003-04$ \\
\hline Diphtheria-tetanus-pertussis-polio-revaccination & $2004-$ \\
\hline 7-valent pneumococcal vaccine & $2007-10$ \\
\hline Vaccine against human papilloma virus types 6, 11, 16, and 18 & $2010-$ \\
\hline 13-valent pneumococcal vaccine (replacing 7-valent pneumococcal vaccine) & 2009- (began in 2008) \\
\hline
\end{tabular}

List updated on 13 September 2011. Hib=Haemophilus influenzae type B. 


\begin{tabular}{|c|c|c|c|}
\hline \multirow[b]{2}{*}{ Age (years) } & \multicolumn{2}{|c|}{ Incidence per 100000 person years $(95 \% \mathrm{Cl})$} & \multirow[b]{2}{*}{ Total } \\
\hline & Female participants & Male participants & \\
\hline \multicolumn{4}{|c|}{ Acute infectious and post-infectious polyneuritis (Guillain-Barré syndrome) } \\
\hline$<1$ & $0.18(0.02$ to 0.64$)$ & $0.00(0.00$ to 0.31$)$ & $0.09(0.01$ to 0.31$)$ \\
\hline $1-3$ & $0.28(0.13$ to 0.53$)$ & $0.80(0.53$ to 1.17$)$ & $0.55(0.38$ to 0.76$)$ \\
\hline 4-9 & $0.55(0.37$ to 0.78$)$ & $0.65(0.46$ to 0.89$)$ & $0.60(0.46$ to 0.76$)$ \\
\hline $10-17$ & $1.00(0.75$ to 1.30$)$ & $0.88(0.65$ to 1.17$)$ & $0.94(0.76$ to 1.14$)$ \\
\hline Total & $0.62(0.50$ to 0.76$)$ & $0.71(0.59$ to 0.86$)$ & $0.67(0.58$ to 0.77$)$ \\
\hline \multicolumn{4}{|c|}{ Acute transverse myelitis } \\
\hline$<1$ & $0.62(0.25$ to 1.29$)$ & 0.34 (0.09 to 0.87$)$ & $0.48(0.24$ to 0.86$)$ \\
\hline $1-3$ & $0.34(0.17$ to 0.61$)$ & $0.39(0.21$ to 0.66$)$ & $0.36(0.23$ to 0.54$)$ \\
\hline $4-9$ & $0.05(0.01$ to 0.16$)$ & $0.16(0.07$ to 0.30$)$ & $0.11(0.06$ to 0.19$)$ \\
\hline $10-17$ & 0.75 (0.54 to 1.02$)$ & $0.45(0.29$ to 0.66$)$ & $0.60(0.46$ to 0.76$)$ \\
\hline Total & 0.40 (0.31 to 0.52$)$ & $0.32(0.24$ to 0.42$)$ & $0.36(0.30$ to 0.44$)$ \\
\hline \multicolumn{4}{|c|}{ Optic polyneuritis } \\
\hline$<1$ & $0.00(0.00$ to 0.33$)$ & $0.00(0.00$ to 0.31$)$ & $0.00(0.00$ to 0.16$)$ \\
\hline $1-3$ & $0.03(0.00$ to 0.17$)$ & 0.03 (0.00 to 0.17$)$ & $0.03(0.00$ to 0.11$)$ \\
\hline $4-9$ & $0.38(0.24$ to 0.59$)$ & 0.31 (0.19 to 0.50$)$ & 0.35 (0.25 to 0.48$)$ \\
\hline $10-17$ & 1.91 (1.56 to 2.32 ) & 0.79 (0.58 to 1.06$)$ & 1.34 (1.13 to 1.58$)$ \\
\hline Total & $0.82(0.68$ to 0.98$)$ & $0.40(0.31$ to 0.51$)$ & $0.60(0.52$ to 0.70$)$ \\
\hline \multicolumn{4}{|c|}{ Facial nerve palsy } \\
\hline$<1$ & 5.88 (4.55 to 7.48$)$ & 8.52 (6.93 to 10.36$)$ & 7.23 (6.17 to 8.42$)$ \\
\hline $1-3$ & 5.18 (4.43 to 6.03$)$ & 5.46 (4.70 to 6.31$)$ & 5.33 (4.78 to 5.92$)$ \\
\hline $4-9$ & $5.62(5.01$ to 6.29$)$ & 5.93 (5.31 to 6.60$)$ & 5.78 (5.34 to 6.24$)$ \\
\hline $10-17$ & $9.92(9.09$ to 10.80$)$ & $8.02(7.29$ to 8.80$)$ & 8.95 (8.40 to 9.53$)$ \\
\hline Total & 7.06 (6.64 to 7.50$)$ & 6.76 (6.36 to 7.17$)$ & 6.91 (6.62 to 7.20$)$ \\
\hline \multicolumn{4}{|c|}{ Anaphylactic shock } \\
\hline$<1$ & $0.53(0.20$ to 1.16$)$ & $0.68(0.29$ to 1.34$)$ & 0.61 (0.33 to 1.02$)$ \\
\hline $1-3$ & $0.65(0.40$ to 1.00$)$ & $1.19(0.85$ to 1.62$)$ & $0.93(0.71$ to 1.19$)$ \\
\hline 4-9 & 0.69 (0.49 to 0.95$)$ & 1.75 (1.42 to 2.13$)$ & 1.23 (1.04 to 1.46$)$ \\
\hline $10-17$ & 1.92 (1.56 to 2.32 ) & 2.43 (2.04 to 2.88 ) & 2.18 (1.91 to 2.47 ) \\
\hline Total & 1.10 (0.94 to 1.28$)$ & 1.79 (1.59 to 2.01$)$ & 1.45 (1.32 to 1.59$)$ \\
\hline \multicolumn{4}{|l|}{ Seizure } \\
\hline$<1$ & 445.83 (433.55 to 458.37 ) & 503.73 (490.96 to 516.75$)$ & 475.43 (466.55 to 484.44$)$ \\
\hline $1-3$ & 537.86 (529.82 to 545.99$)$ & $646.84(638.20$ to 655.56$)$ & 593.49 (587.57 to 599.45$)$ \\
\hline $4-9$ & 39.99 (38.32 to 41.72$)$ & 54.21 (52.30 to 56.18 ) & 47.24 (45.96 to 48.55$)$ \\
\hline $10-17$ & 30.98 (29.49 to 32.52$)$ & $30.69(29.24$ to 32.19$)$ & 30.83 (29.79 to 31.90$)$ \\
\hline Total & 173.05 (170.95 to 175.17$)$ & 205.92 (203.67 to 208.19) & $189.82(188.27$ to 191.37$)$ \\
\hline \multicolumn{4}{|c|}{ Multiple sclerosis } \\
\hline$<1$ & 4.90 (3.69 to 6.38$)$ & $6.48(5.10$ to 8.10$)$ & 5.71 (4.77 to 6.77 ) \\
\hline $1-3$ & 5.96 (5.15 to 6.87$)$ & 10.13 (9.08 to 11.26$)$ & 8.09 (7.42 to 8.81$)$ \\
\hline 4-9 & 3.00 (2.55 to 3.49$)$ & 4.83 (4.28 to 5.43$)$ & 3.93 (3.57 to 4.32 ) \\
\hline $10-17$ & 1.92 (1.56 to 2.33$)$ & 1.51 (1.21 to 1.87$)$ & 1.71 (1.47 to 1.98$)$ \\
\hline Total & 3.39 (3.10 to 3.69$)$ & $4.92(4.58$ to 5.27$)$ & 4.17 (3.94 to 4.40$)$ \\
\hline \multicolumn{4}{|c|}{ Autoimmune thrombocytopenia } \\
\hline$<1$ & $0.18(0.02$ to 0.64$)$ & 0.94 (0.47 to 1.68$)$ & $0.57(0.30$ to 0.97$)$ \\
\hline $1-3$ & 0.50 (0.28 to 0.81$)$ & $0.74(0.48$ to 1.10$)$ & $0.62(0.45$ to 0.84$)$ \\
\hline $4-9$ & $0.29(0.17$ to 0.47$)$ & $0.28(0.16$ to 0.45$)$ & $0.29(0.20$ to 0.40$)$ \\
\hline $10-17$ & $0.15(0.06$ to 0.30$)$ & $0.11(0.04$ to 0.24$)$ & $0.13(0.07$ to 0.22$)$ \\
\hline
\end{tabular}


Table 3 (continued)

\begin{tabular}{|c|c|c|c|}
\hline \multirow[b]{2}{*}{ Age (years) } & \multicolumn{2}{|c|}{ Incidence per 100000 person years $(95 \% \mathrm{Cl})$} & \multirow[b]{2}{*}{ Total } \\
\hline & Female participants & Male participants & \\
\hline Total & $0.28(0.20$ to 0.37$)$ & $0.37(0.28$ to 0.47$)$ & $0.32(0.26$ to 0.39$)$ \\
\hline \multicolumn{4}{|c|}{ Type 1 diabetes mellitus } \\
\hline$<1$ & 3.92 (2.85 to 5.26$)$ & 4.86 (3.68 to 6.29$)$ & 4.40 (3.58 to 5.35$)$ \\
\hline $1-3$ & 8.97 (7.97 to 10.07$)$ & 9.98 (8.94 to 11.10$)$ & 9.49 (8.76 to 10.26$)$ \\
\hline 4-9 & $17.78(16.68$ to 18.93$)$ & $17.18(16.12$ to 18.29$)$ & $17.47(16.71$ to 18.26$)$ \\
\hline $10-17$ & 23.98 (22.69 to 25.34$)$ & $27.48(26.12$ to 28.90$)$ & 25.77 (24.82 to 26.74$)$ \\
\hline Total & $17.06(16.40$ to 17.73$)$ & 18.34 (17.68 to 19.02$)$ & $17.71(17.25$ to 18.19$)$ \\
\hline
\end{tabular}

Juvenile and rheumatoid arthritis

\begin{tabular}{|c|c|c|c|}
\hline$<1$ & 2.58 (1.73 to 3.71$)$ & 2.22 (1.45 to 3.25$)$ & 2.40 (1.80 to 3.12$)$ \\
\hline $1-3$ & 24.50 (22.82 to 26.27$)$ & 13.01 (11.82 to 14.28$)$ & $18.63(17.60$ to 19.70$)$ \\
\hline 4-9 & $15.96(14.91$ to 17.05$)$ & $14.80(13.82$ to 15.83$)$ & $15.37(14.65$ to 16.11$)$ \\
\hline $10-17$ & 26.25 (24.89 to 27.66$)$ & $14.07(13.10$ to 15.09$)$ & 20.03 (19.20 to 20.89$)$ \\
\hline Total & 20.40 (19.69 to 21.13$)$ & $13.23(12.67$ to 13.81$)$ & $16.73(16.28$ to 17.20$)$ \\
\hline \multicolumn{4}{|c|}{ Narcolepsy } \\
\hline$<1$ & $0.00(0.00$ to 0.33$)$ & $0.00(0.00$ to 0.31$)$ & $0.00(0.00$ to 0.16$)$ \\
\hline $1-3$ & 0.09 (0.02 to 0.27$)$ & $0.18(0.07$ to 0.39$)$ & $0.14(0.06$ to 0.26$)$ \\
\hline 4-9 & $0.27(0.15$ to 0.45$)$ & 0.42 (0.27 to 0.62$)$ & 0.35 (0.25 to 0.48$)$ \\
\hline $10-17$ & 0.88 (0.65 to 1.17$)$ & 0.97 (0.73 to 1.27$)$ & $0.93(0.76$ to 1.13$)$ \\
\hline Total & $0.43(0.33$ to 0.55$)$ & $0.53(0.42$ to 0.66$)$ & $0.48(0.41$ to 0.56$)$ \\
\hline \multicolumn{4}{|c|}{ Death of unknown cause } \\
\hline$<1$ & 13.99 (11.89 to 16.36$)$ & $17.55(15.24$ to 20.12$)$ & $15.81(14.23$ to 17.53$)$ \\
\hline $1-3$ & 1.49 (1.10 to 1.98$)$ & $1.84(1.41$ to 2.36$)$ & $1.67(1.37$ to 2.01$)$ \\
\hline 4-9 & 0.27 (0.15 to 0.45$)$ & $0.35(0.21$ to 0.54$)$ & $0.31(0.22$ to 0.43$)$ \\
\hline $10-17$ & $0.26(0.14$ to 0.44$)$ & $0.76(0.55$ to 1.02$)$ & 0.51 (0.39 to 0.67$)$ \\
\hline Total & $1.54(1.35$ to 1.76$)$ & 2.09 (1.87 to 2.32$)$ & $1.82(1.67$ to 1.98$)$ \\
\hline
\end{tabular}

\section{Any adverse event}

$<1 \quad 478.36$ (465.64 to 491.34 ) 545.20 (531.92 to 558.74 ) 512.53 (503.31 to 521.88 )

$1-3 \quad 584.82$ (576.43 to 593.30$) 689.30$ (680.38 to 698.31$) 638.16$ (632.02 to 644.34$)$

\begin{tabular}{llll}
\hline $4-9$ & $83.49(81.06$ to 85.97$)$ & $99.46(96.86$ to 102.11$)$ & $91.63(89.85$ to 93.44$)$ \\
\hline
\end{tabular}

$10-17 \quad 96.73$ (94.08 to 99.43$) \quad 85.15$ (82.71 to 87.64$) \quad 90.82$ (89.03 to 92.65$)$

Total 224.63 (222.23 to 227.05$) 253.94$ (251.44 to 256.47) 239.58 (237.85 to 241.33)

Any adverse event excluding seizure

\begin{tabular}{llll}
\hline$<1$ & $31.02(27.84$ to 34.45$)$ & $40.05(36.51$ to 43.84$)$ & $35.64(33.24$ to 38.16$)$ \\
\hline $1-3$ & $45.39(43.09$ to 47.78$)$ & $40.95(38.82$ to 43.17$)$ & $43.12(41.55$ to 44.74$)$ \\
\hline $4-9$ & $42.70(40.99$ to 44.47$)$ & $44.03(42.33$ to 45.79$)$ & $43.38(42.17$ to 44.62$)$ \\
\hline $10-17$ & $65.04(62.88$ to 67.24$)$ & $53.88(51.96$ to 55.85$)$ & $59.34(57.90$ to 60.81$)$ \\
\hline Total & $50.24(49.12$ to 51.38$)$ & $46.53(45.47$ to 47.60$)$ & $48.34(47.57$ to 49.12$)$ \\
\hline
\end{tabular}


Table 4| Predicted number of outcomes temporally associated with a vaccine dose

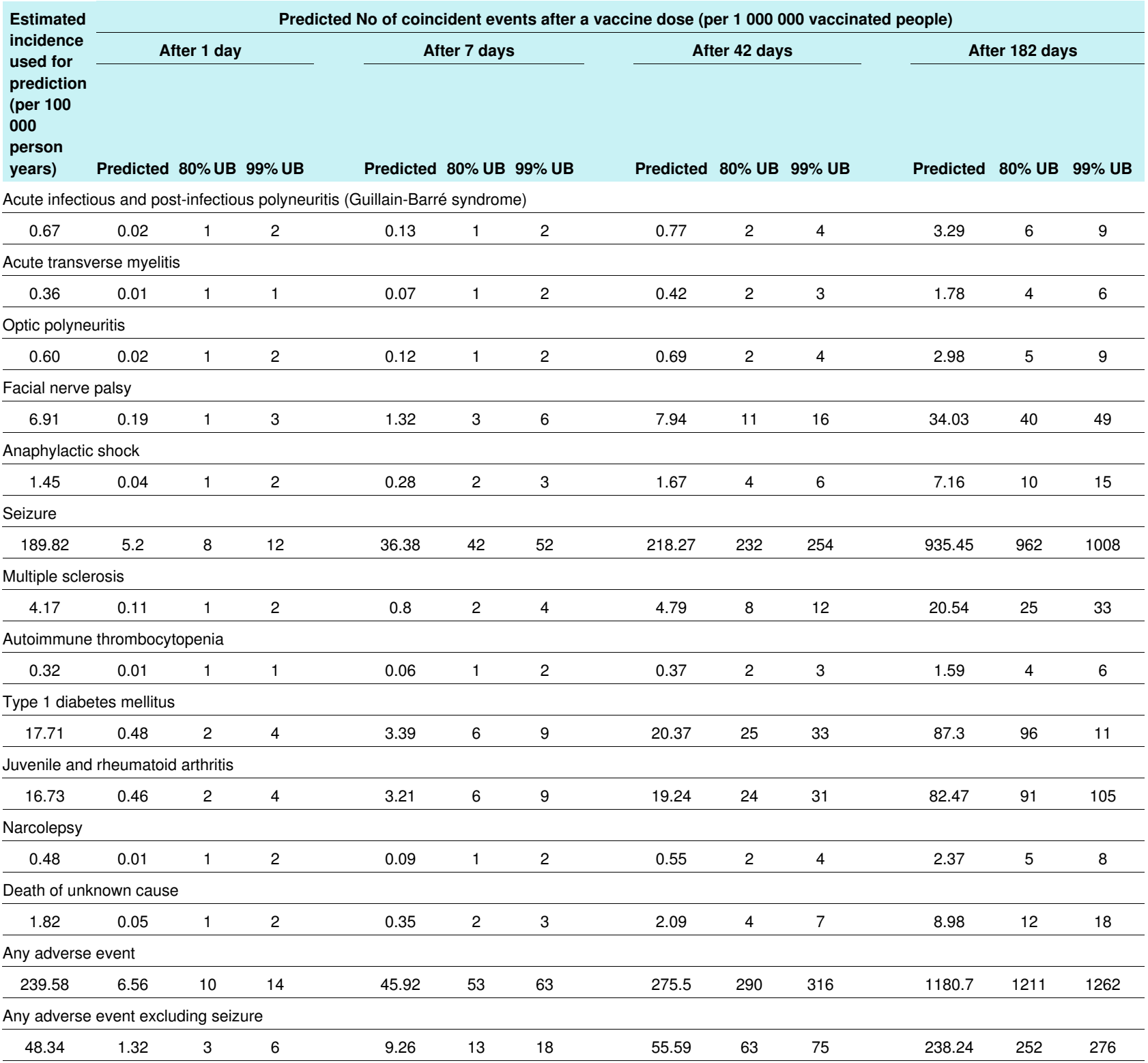

UB=upper boundary ( $80 \%$ and $99 \%$ upper boundaries of the predicted number of coincident adverse events can assess the likelihood that adverse events of a given type are not only temporally, but also causally associated with a vaccine dose). 


\section{Figures}
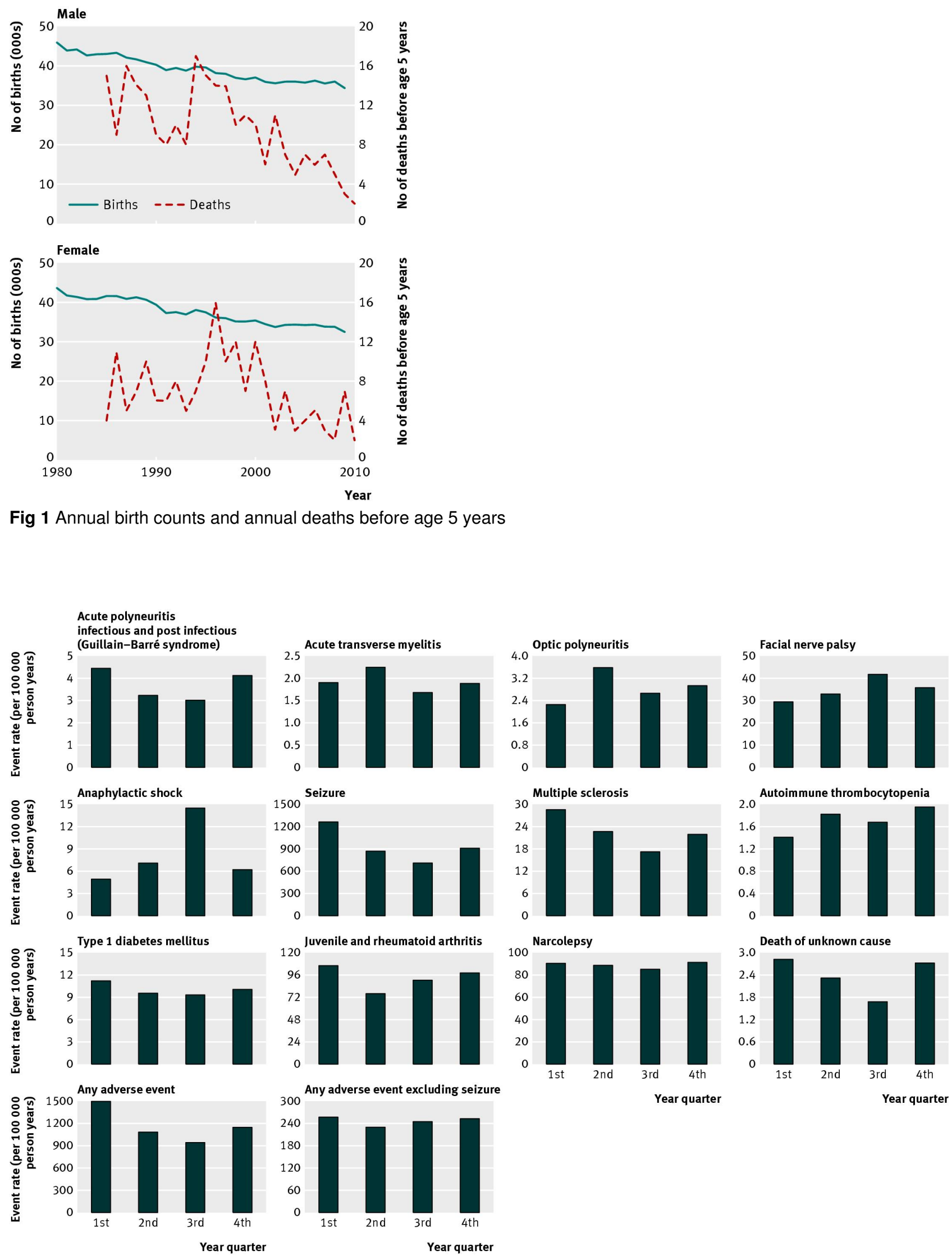

Fig 2 Seasonal distribution of incidence (per 100000 person years) of selected outcome diagnoses. Data are shown for each quarter of the year 